\title{
Food additives such as sodium sulphite, sodium benzoate and curcumin inhibit leptin release in lipopolysaccharide-treated murine adipocytes in vitro
}

\author{
Christian Ciardi ${ }^{1}$, Marcel Jenny ${ }^{2}$, Alexander Tschoner ${ }^{1}$, Florian Ueberall ${ }^{3}$, Josef Patsch ${ }^{1}$, \\ Michael Pedrini ${ }^{1}$, Christoph Ebenbichler ${ }^{1}$ and Dietmar Fuchs ${ }^{2 *}$ \\ ${ }^{1}$ Department of Internal Medicine I, Anichstrasse 35, Innsbruck Medical University, A-6020 Innsbruck, Austria \\ ${ }^{2}$ Division of Biological Chemistry, Biocenter, Fritz-Pregl-Straße 3, Innsbruck Medical University, A-6020 Innsbruck, Austria \\ ${ }^{3}$ Division of Medical Biochemistry, Biocenter, Fritz-Pregl-Straße 3, Innsbruck Medical University, A-6020 Innsbruck, Austria
}

(Received 7 January 2011 - Revised 7 June 2011 - Accepted 7 June 2011 - First published online 1 August 2011)

\begin{abstract}
Obesity leads to the activation of pro-inflammatory pathways, resulting in a state of low-grade inflammation. Recently, several studies have shown that the exposure to lipopolysaccharide (LPS) could initiate and maintain a chronic state of low-grade inflammation in obese people. As the daily intake of food additives has increased substantially, the aim of the present study was to investigate a potential influence of food additives on the release of leptin, IL- 6 and nitrite in the presence of LPS in murine adipocytes. Leptin, IL-6 and nitrite concentrations were analysed in the supernatants of murine 3T3-L1 adipocytes after co-incubation with LPS and the food preservatives, sodium sulphite (SS), sodium benzoate (SB) and the spice and colourant, curcumin, for $24 \mathrm{~h}$. In addition, the kinetics of leptin secretion was analysed. A significant and dose-dependent decrease in leptin was observed after incubating the cells with SB and curcumin for 12 and $24 \mathrm{~h}$, whereas SS decreased leptin concentrations after $24 \mathrm{~h}$ of treatment. Moreover, SS increased, while curcumin decreased LPS-stimulated secretion of IL-6, whereas SB had no such effect. None of the compounds that were investigated influenced nitrite production. The food additives SS, SB and curcumin affect the leptin release after co-incubation with LPS from cultured adipocytes in a dose- and timedependent manner. Decreased leptin release during the consumption of nutrition-derived food additives could decrease the amount of circulating leptin to which the central nervous system is exposed and may therefore contribute to an obesogenic environment.
\end{abstract}

Key words: Adipocytes: Adipokines: Antioxidants: Leptin

Changes in lifestyle including overnutrition and physical inactivity have led to a rise of excess body weight during the last decades. Severe obesity is the sixth most important risk factor contributing to the overall burden of diseases worldwide due to the main adverse consequences such as CVD, metabolic disorders and several types of cancer ${ }^{(1)}$. It is well recognised that obesity is associated with a state of chronic inflammation due to different pathogenic mechanisms ${ }^{(2)}$. Lipid accumulation leads to adipocyte hypertrophy, cellular stress, increased lipolysis and activation of pro-inflammatory pathways, resulting in an increased production and secretion of pro-inflammatory cytokines by adipocytes and macrophages $^{(3)}$. Moreover, new evidence supports the idea that some key aspects of the mammalian host-gut microbial relationship could play a major role in obesity. LPS is continuously produced within the gut by the death of Gram-negative bacteria and is absorbed into the intestinal capillaries to be transported by lipoproteins leading to a state of metabolic endotoxaemia, which seems to be a potential pathway to initiating and maintaining a state of low-grade inflammation associated with obesity ${ }^{(4-6)}$.

Among the inflammation-related cytokines, adipocytes secrete various adipokines, which play a major role in energy homeostasis by exerting multiple favourable effects on lipid and carbohydrate metabolism ${ }^{(7)}$. It is recognised that these beneficial effects are removed in states of severe obesity. Leptin, which is produced in proportion to fat stores, plays a crucial role in the regulation of appetite, food intake and energy homeostasis by signalling the information of available energy within the central nervous system ${ }^{(8-10)}$. Various studies have investigated a potential effect of food composition on circulating leptin levels over the last years ${ }^{(11-13)}$. However, studies analysing the influence of food preservatives, which are widely used to preserve aliments, are scarce.

The addition of food preservatives such as antioxidants and food colourants prevents the growth of bacteria, fungi and

Abbreviations: LPS, lipopolysaccharide; SS, sodium sulphite; SB, sodium benzoate.

*Corresponding author: D. Fuchs, fax +43 5129003 73330, email dietmar.fuchs@i-med.ac.at 
other micro-organisms. Furthermore, these additives decelerate oxidation of fats preventing rancidity, and inhibit ageing and discolouration of food. The daily intake of food has increased substantially and is responsible for the dramatic rise of the prevalence of obesity inducing metabolic disorders such as insulin resistance, diabetes mellitus, inflammation and blood lipid disorders ${ }^{(1,14)}$. As a consequence of increased food ingestion, the intake of antioxidant food supplements has increased exponentially.

The aim of the present study was to analyse the effect of the widely used food preservatives sodium sulphite (E221, SS), sodium benzoate (E211, SB) and the spice and food colourant curcumin (E100) on the leptin release of unstimulated and LPS-stimulated adipocytes, in order to investigate a potential contribution of these diet-derived agents to the development of obesity-related metabolic perturbations.

\section{Experimental methods}

\section{Cell culture}

Murine 3T3-L1 fibroblasts cells were obtained from the American Type Culture Collection (ATCC-CL-173; Manassas, VA, USA) and cultured in $5 \% \mathrm{CO}_{2}$ at $37^{\circ} \mathrm{C}$. The cells were maintained in Dulbecco's modified Eagle's medium (GIBCO, Karlsruhe, Germany) supplemented with $5 \mathrm{~mm}$-glucose, $10 \%$ heat-inactivated bovine serum, $2 \mathrm{~mm}$-L-glutamine, $100 \mathrm{U} / \mathrm{ml}$ penicillin and $100 \mu \mathrm{g} / \mathrm{ml}$ streptomycin. The medium was changed every $2 \mathrm{~d}$. At $2 \mathrm{~d}$ after reaching confluence, the pre-adipocytes were treated with a medium to induce differentiation as described previously ${ }^{(15)}$. In short, $2 \mathrm{~d}$ post-confluence cultured cells were supplemented with $0.5 \mathrm{~mm}$-1-methyl-3isobutylmethylxanthine $+1.0 \mathrm{~mm}$-dexamethasone $+10 \mu \mathrm{g} / \mathrm{ml}$ insulin $+10 \%$ fetal bovine serum for another $2 \mathrm{~d}$. Then the cells were kept in a culture medium with $10 \mu \mathrm{g} / \mathrm{ml}$ insulin $+10 \%$ fetal bovine serum for another $2 \mathrm{~d}$. After differentiation, the culture medium was replaced every 2nd day using a culture medium with $10 \%$ fetal bovine serum. As determined by light microscopy, over $90 \%$ of the cells that were used for the experiments were differentiated. All experiments were performed within $10-14 \mathrm{~d}$ postdifferentiation.

\section{Experimental procedures}

First, the adipocytes were incubated with increasing concentrations of SS, SB and curcumin for $24 \mathrm{~h}$ to analyse a possible dose-dependent effect on leptin secretion. Accordingly, the cells were washed twice with PBS and incubated with $1 \mathrm{~mm}-$ and $10 \mathrm{~mm}-\mathrm{SS}, 10 \mathrm{~mm}-$ and $20 \mathrm{~mm}-\mathrm{SB}$, or $10 \mu \mathrm{m}-$ and $50 \mu \mathrm{m}-$ curcumin.

After the incubation of cells with food additives, we stimulated the adipocytes with $1 \mu \mathrm{g} / \mathrm{ml}$ LPS (Sigma-Aldrich, Munich, Germany) and incubated for $24 \mathrm{~h}$ before the measurement of leptin for the dose-response experiments. To assess the effect of LPS on the secretion of pro-inflammatory cytokines, IL-6 concentrations were measured in the cell culture supernatant. Furthermore, the concentration of the nitrite in the cell culture supernatant was determined, which represents a stable end product of nitrite oxide and thus an estimate of NO synthase activity.

Finally, time-course experiments were conducted using $1 \mathrm{~mm}$-SS, $10 \mathrm{~mm}$-SB and $50 \mu \mathrm{m}$-curcumin. We incubated the cells for 6, 12 and $24 \mathrm{~h}$ with and without LPS, and measured the leptin levels in the supernatant. All the experiments were replicated three times in triplicates.

\section{Cell viability}

To measure the possible effects of the food additives or LPS on the viability of 3T3-L1 adipocytes during the experiments, we used the lactate dehydrogenase release assay. Lactate dehydrogenase was measured in the cell culture supernatant by using an autoanalyser (ABX-Cobas Mira; Roche Diagnostic, Mannheim, Germany) at the beginning and at the end of all incubations according to the manufacturer's instructions. Lactate dehydrogenase is an ubiquitous, intracellular-located enzyme, which is detectable in the cell culture supernatant only after cell lysis due to cytotoxicity.

\section{Analysis of leptin secretion}

Leptin concentrations were measured in the cell culture supernatant using an ELISA kit for mouse leptin from Research and Diagnostic Systems (Quantikine ${ }^{\circledR}$ M Murine; R\&D Systems, Minneapolis, MN, USA; inter-assay CV\% $<5$; intraassay $\mathrm{CV} \%<3 \cdot 8)$.

\section{Analysis of IL-6 secretion}

IL-6 concentrations were determined in the cell culture supernatant using an ELISA kit for mouse IL-6 (Quantikine ${ }^{\circledR}$ Mouse IL-6; R\&D Systems; inter-assay CV\% $<7 \cdot 6$; intra-assay $\mathrm{CV} \%<3.5)$.

\section{Analysis of nitrite}

Nitrite concentrations in cellular supernatants were determined photometrically using the Griess reaction. Thereby, nitrite in the samples was quantitatively converted to a diazonium salt, which was then coupled with $N(1$-naphthyl) ethylenediamine dihydrochloride, forming an azo dye that was read at $540 \mathrm{~nm}$ in a spectrophotometer.

\section{Statistical analysis}

Data are expressed as means and standard deviations unless otherwise indicated. Normality of data was assessed using the Shapiro-Wilk test. Differences between groups were analysed by one-way ANOVA. Post hoc adjustment for multiple comparisons was applied according to the method of Bonferroni.

For the comparison of mean values within the groups during the time-course experiment, one-way ANOVA for repeated measures was used. In the case of significant differences, Bonferroni's post hoc tests were performed. The 
Greenhouse-Geisser correction was applied when indicated by Mauchly's test for sphericity. $P$ values $\leq 0.05$ were considered statistically significant.

All analyses were performed using SPSS 15.0 for Windows (SPSS, Chicago, IL, USA).

\section{Results}

\section{Effect of lipopolysaccharide and toxicity tests}

At $10 \mathrm{~d}$ after the initiation of differentiation of murine 3T3-L1 fibroblasts into adipocytes, the cells secreted a significant amount of leptin into the cell culture supernatant. In all the experiments, we used untreated cells as a negative control and LPS $(1 \mu \mathrm{g} / \mathrm{ml})$-stimulated cells as a positive control. The treatment of adipocytes with LPS for $24 \mathrm{~h}$ led to a significant diminution of leptin concentrations by approximately $30 \%$ and a significant increase in IL-6 secretion by nearly $800 \%$ compared with control cells in the absence of LPS. By the measurements of lactate dehydrogenase in the cell culture supernatants at the beginning and at the end of all incubation procedures, we did not observe any influence of LPS or the food additives on cell viability (data not shown).

First, we analysed the release of leptin, IL-6 and nitrite after the treatment of unstimulated and LPS-stimulated adipocytes with SS, SB and curcumin at different concentrations for $24 \mathrm{~h}$. The incubation of cells with food additives in the absence of LPS did not affect leptin levels in the supernatants of adipocyte cultures (data not shown).

The antioxidant, sodium sulphite, reduces leptin release after co-incubation with lipopolysaccharide

Co-incubation of cells with LPS and $1 \mathrm{~mm}-\mathrm{SS}$ induced a stronger decrease in leptin concentrations by $30 \%$ compared with the LPS-stimulated control in the absence of this food additive ( $P \leq 0 \cdot 001$; Fig. 1(a)). Increasing the SS concentration to $10 \mathrm{~mm}$ did not further decrease leptin concentrations significantly. However, a significant increase of $57 \%$ of IL- 6 was found by augmenting the concentration of SS $(P<0 \cdot 01$; Fig. 1(b)). With regard to nitrite formation, we found no effect after treatment with SS (Fig. 1(c)).

\section{Sodium benzoate decreases leptin release after co-incubation with lipopolysaccharide}

Co-treatment of LPS-stimulated adipocytes with $10 \mathrm{~mm}-\mathrm{SB}$ decreased leptin levels by $49 \%(P<0 \cdot 001$; Fig. 2(a)), which was even more pronounced by increasing the concentration of $\mathrm{SB}$ to $20 \mathrm{~mm}(-70 \% ; P<0.001$; Fig. 2(a)). No significant effects could be detected on IL- 6 and nitrite concentrations after the incubation of LPS-stimulated cells with SB (Fig. 2(b) and (c))
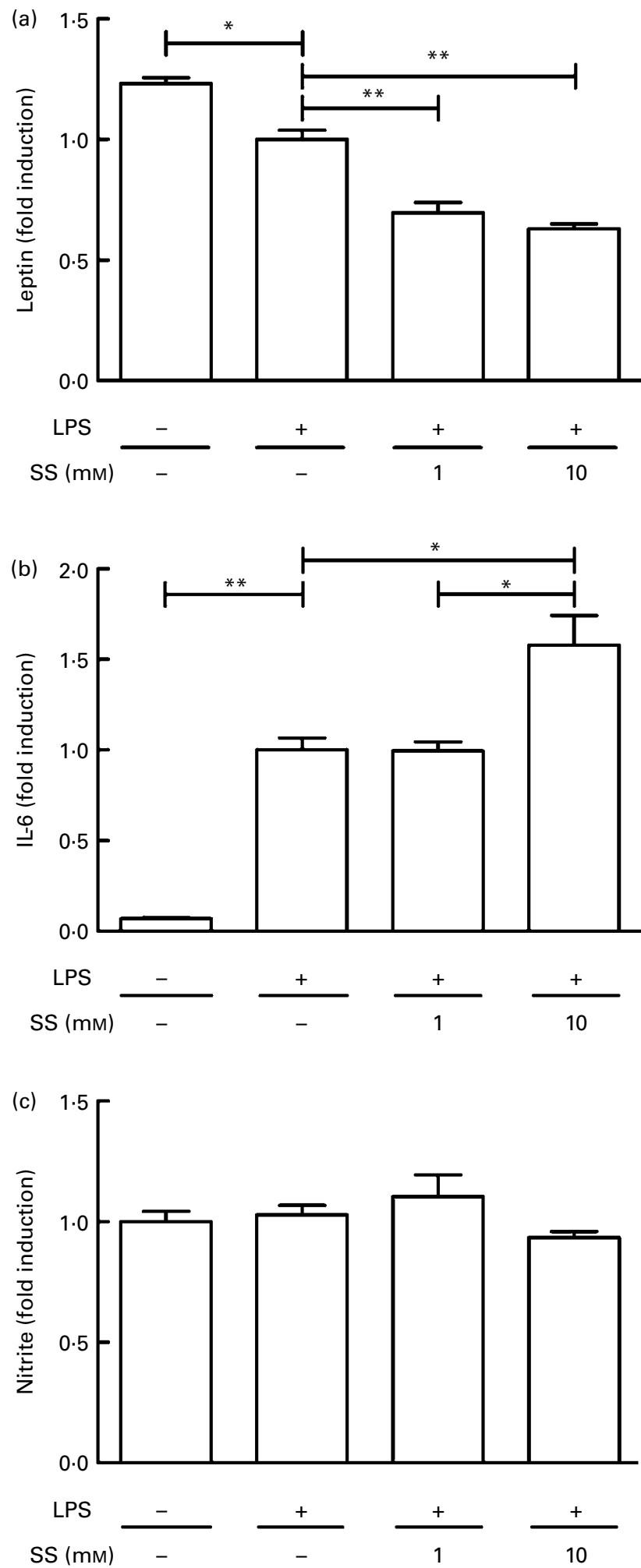

Fig. 1. Influence of sodium sulphite (SS) in lipopolysaccharide (LPS; $1 \mu \mathrm{g} / \mathrm{ml}$ )-treated 3T3-L1 cells on (a) leptin secretion, (b) IL-6 release and (c) NO formation after $24 \mathrm{~h}$ of treatment. The control conditions in the absence or presence of $1 \mu \mathrm{g} / \mathrm{ml}$ LPS are shown. Fold induction was related to the control group with LPS in the absence of SS. All experiments were performed in triplicates. Values are means, with their standard errors represented by vertical bars. Mean values were significantly different as assessed by one-way ANOVA with Bonferroni's adjustment: ${ }^{*} P \leq 0.01$ and ${ }^{\star *} P \leq 0.001$. 

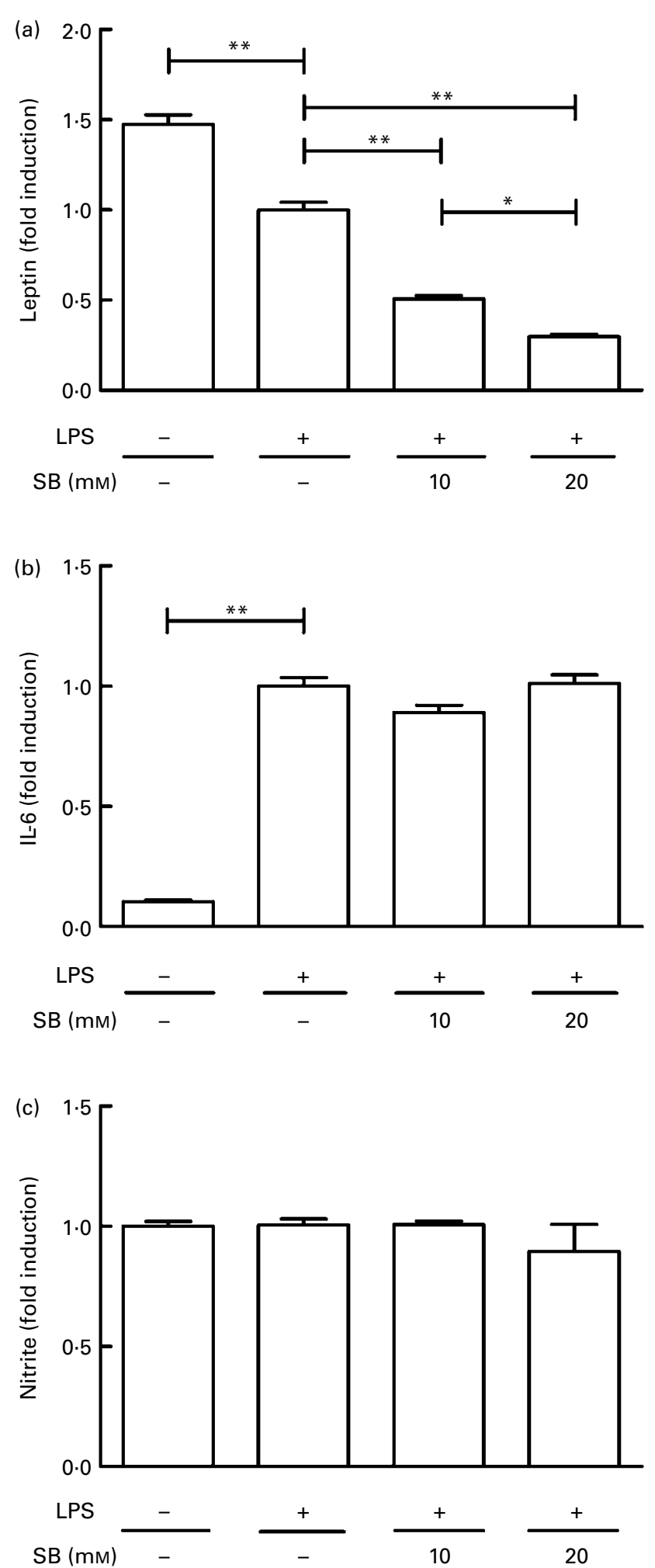

Fig. 2. Influence of sodium benzoate (SB) in lipopolysaccharide (LPS; $1 \mu \mathrm{g} / \mathrm{ml}$ )-treated 3T3-L1 cells on (a) leptin secretion, (b) IL-6 release and (c) NO formation after $24 \mathrm{~h}$ of treatment. The control conditions in the absence or presence of $1 \mu \mathrm{g} / \mathrm{ml}$ LPS are shown. Fold induction was related to the control group with LPS in the absence of SB. All experiments were performed in triplicates. Values are means, with their standard errors represented by vertical bars. Mean values were significantly different as assessed by one-way ANOVA with Bonferroni's adjustment: ${ }^{*} P \leq 0.01$ and ${ }^{\star *} P \leq 0.001$.
The food colourant and antioxidant curcumin decreases leptin secretion after co-incubation with lipopolysaccharide

A significant decrease in leptin release by $18 \%$ was detected after the incubation of the cells with $10 \mu \mathrm{m}$-curcumin $(P<0.01$; Fig. 3(a)), which was further suppressed with $50 \mu \mathrm{M}$ by $87 \%(P<0.001$; Fig. 3(a)). In contrast to SS and SB, co-treatment of LPS-stimulated adipocytes with $50 \mu \mathrm{M}$-curcumin significantly decreased IL-6 concentrations by $57 \%$ in the supernatant of the cells (Fig. 3(b)). Again, no effect of curcumin was detected on the formation of nitrite (Fig. 3(c)).

\section{Antioxidants influence leptin secretion over time}

Next, we investigated the effect of food additives on leptin secretion into the cell culture supernatant as a function of time (Fig. 4). In unstimulated cells, leptin concentrations rose from 49.6 (SD 28.3$) \mathrm{pg} / \mathrm{ml}$ after $6 \mathrm{~h}$ to 185.7 (SD $55 \cdot 1) \mathrm{pg} / \mathrm{ml}$ after $12 \mathrm{~h}$, and to 854.4 (SD 203) $\mathrm{pg} / \mathrm{ml}$ after $24 \mathrm{~h}$ of treatment (all $P<0.001 v$. $6 \mathrm{~h}$ ). Incubation of the cells with LPS diminished leptin levels significantly after 12 and $24 \mathrm{~h}$ of treatment to $115 \cdot 5$ (SD 40.9) pg/ml and $529 \cdot 2$ (SD 138.9 ) $\mathrm{pg} / \mathrm{ml}$, respectively (all $P<0.01$ when compared with unstimulated cells). After $6 \mathrm{~h}$ of treatment, no significant changes in leptin levels could be detected on treatment with LPS alone or in combination with the food additives. Co-treatment of LPS-stimulated cells with SS further diminished leptin levels slightly but significantly in relationship with treated cells with LPS after $24 \mathrm{~h}$ of treatment $(P<0.05$; Fig. 4(a)). In contrast, co-treatment of cells with SB or curcumin resulted in a substantial suppression of leptin release into the supernatant after 12 and $24 \mathrm{~h}$ compared with LPS-stimulated adipocytes (Fig. 4(b) and (c)).

\section{Discussion}

In the present study, the food preservatives SS and SB as well as the food colourant, curcumin, diminished leptin production in the cell culture supernatants of LPS-treated murine adipocytes in a dose- and time-dependent fashion. All tested compounds possessed antioxidant and/or radical-scavenging properties, which could play a role in interfering with the signal transduction cascades that modulate leptin production. The function of leptin was originally perceived as a signal that prevented obesity, since leptin-deficient ob/ob mice and leptin-resistant $\mathrm{db} / \mathrm{db}$ mice develop hyperphagia and, consequently, severe obesity ${ }^{(8,16)}$. Supplementation of leptin to genetically deficient ob/ob mice increases their metabolic rate, body temperature and general activity, and decreases food intake, body weight and adiposity ${ }^{(8,17-19)}$. Basal or fasting leptin levels are strongly correlated with adipose tissue mass, percentage of body fat or BMI in both healthy adults and those with type 2 diabetes mellitus ${ }^{(12,20,21-23)}$. Despite these strong correlations, people with similar degrees of adiposity have circulating leptin concentrations that vary considerably, due to the influence of several factors on leptin metabolism such as insulin, glucocorticoids and 

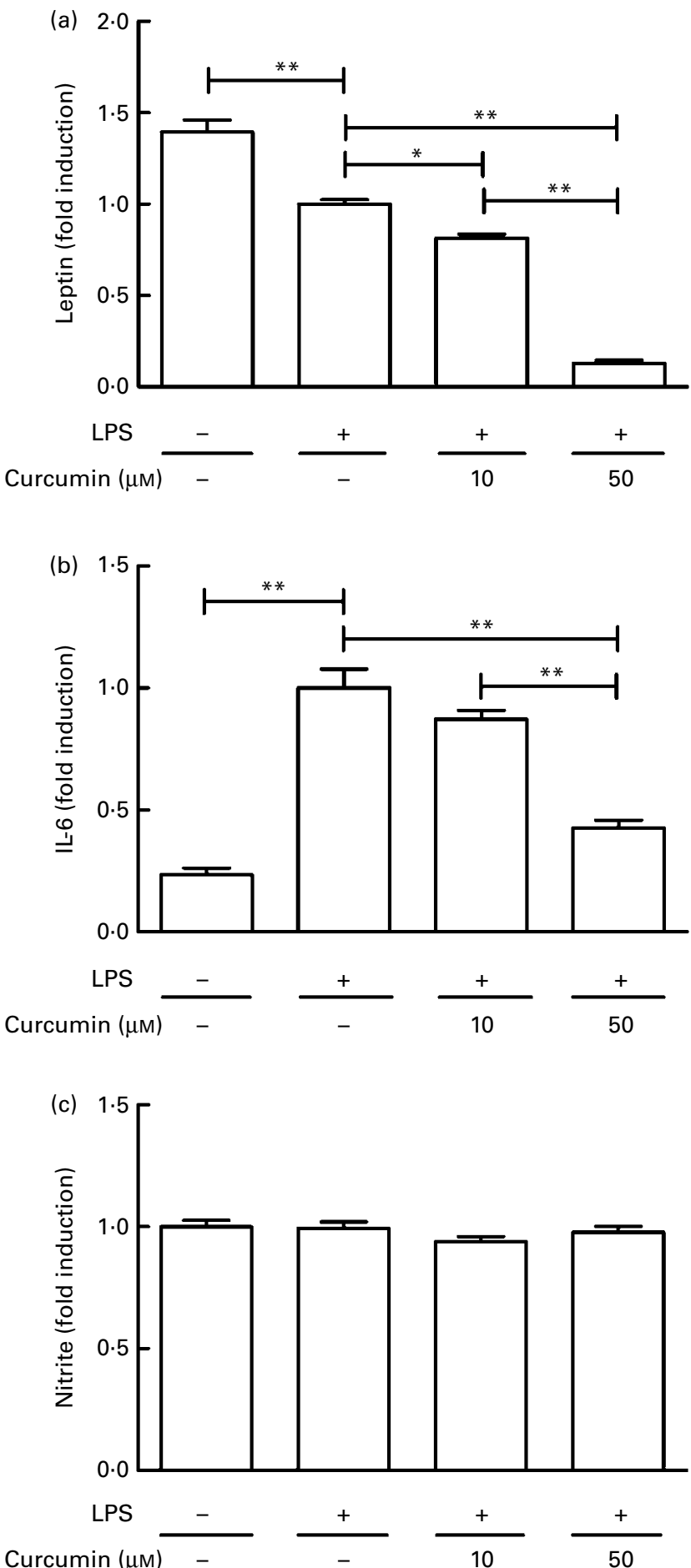

Curcumin $(\mu \mathrm{M})$

10

50

Fig. 3. Influence of curcumin in lipopolysaccharide (LPS; $1 \mu \mathrm{g} / \mathrm{ml}$ )-treated 3T3-L1 cells on (a) leptin secretion, (b) IL-6 release and (c) NO formation after $24 \mathrm{~h}$ of treatment. The control conditions in the absence or presence of $1 \mu \mathrm{g} / \mathrm{ml}$ LPS are shown. Fold induction was related to the control group with LPS in the absence of curcumin. All experiments were performed in triplicates. Values are means, with their standard errors represented by vertical bars. Mean values were significantly different as assessed by oneway ANOVA with Bonferroni's adjustment: ${ }^{\star} P \leq 0.01$ and ${ }^{* *} P \leq 0.001$.
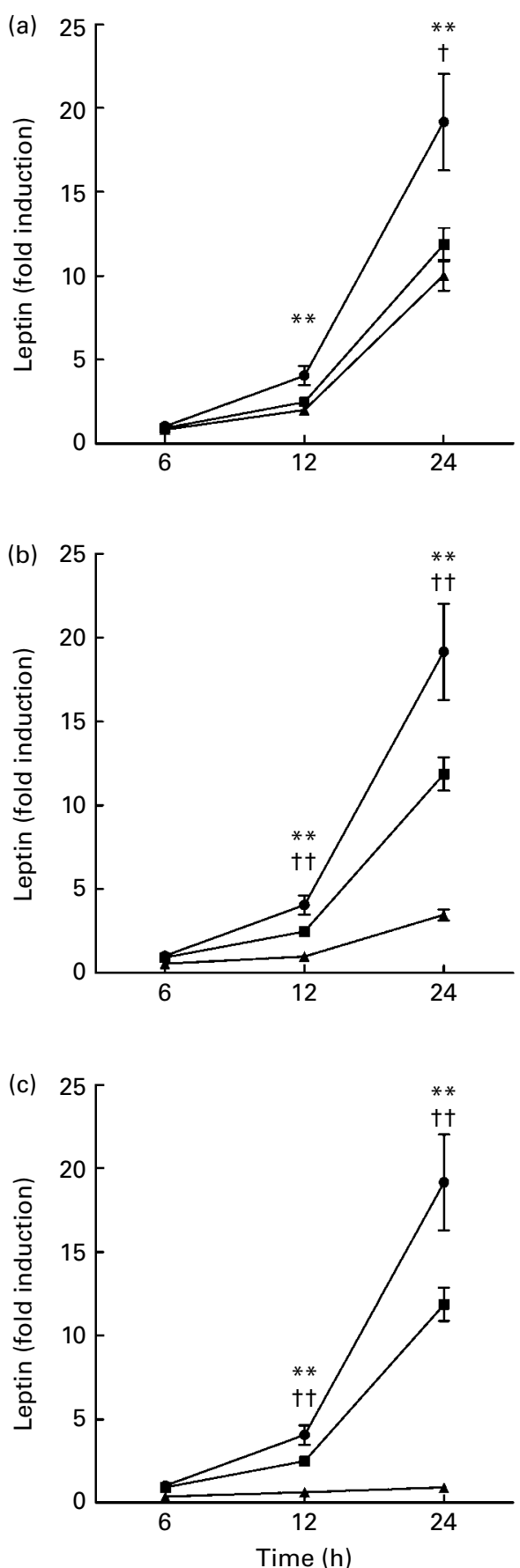

Fig. 4. Time course of leptin production in unstimulated and lipopolysaccharide (LPS; $1 \mu \mathrm{g} / \mathrm{ml}$ )-stimulated 3T3-L1 cells, and in cultures co-incubatedwith LPS $(1 \mu \mathrm{g} / \mathrm{ml})$ and (a) $1 \mathrm{~mm}$-sodium sulphite $(-\boldsymbol{\Delta}-)$, control group - LPS (-๑-), control group + LPS (-口-); (b) $10 \mathrm{mm-sodium}$ benzoate $(-\mathbf{\Lambda}-)$, control group - LPS $(-\bullet-)$, control group + LPS (-口-) or (c) $50 \mu \mathrm{M}$-curcumin $(-\boldsymbol{\Lambda}-)$, control group (--)), control group + LPS $(-\mathbf{-})$. Experiments $\left(\begin{array}{ll}n & 3\end{array}\right)$ were performed in triplicates. Values are means, with their standard errors represented by vertical bars. ${ }^{\star *}$ Mean values were significantly different from those of leptin levels at $6 \mathrm{~h}$ to levels at 12 and $24 \mathrm{~h}$ in the presence of LPS and food additives within the condition as determined by one-way repeated-measures ANOVA, Bonferroni's method applied $(P<0.001)$. Mean values were significantly different of leptin concentration after incubation with LPS in the presence of food preservatives when compared with the control in the presence of LPS alone as determined by one-way ANOVA with Bonferroni's adjustment: $\dagger P<0.05$ and $+\dagger P<0.01$. 
catecholamines, suggesting that leptin metabolism is regulated by a complex network ${ }^{(24)}$.

Based on the current data, different mechanisms induce and maintain a chronic state of inflammation in obese people ${ }^{(2)}$. Among others, it could be shown that in obese states the amount of Gram-negative bacteria differs compared with that in lean individuals. Moreover, due to the increased decay of Gram-negative bacteria, the release of LPS and its absorption in the gut capillaries may induce metabolic endotoxaemia and support chronic inflammation ${ }^{(4-6)}$.

In our model, we found no direct effect on leptin secretion after incubation with antioxidants in the absence of LPS, at low levels of IL-6. However, at high levels of IL-6 due to LPS co-incubation, leptin secretion was significantly less than in the absence of antioxidants. These results suggest that a state of inflammation may be a prerequisite for the observed effect on leptin secretion. In the past 30 years, SS has become one of the leading food preservatives in the food sector throughout the world. The measured concentrations of SS in food vary between $0.8 \mathrm{~mm}$, i.e. in dried potatoes, and $1.6 \mathrm{~mm}$, i.e. in wine and dried fruits ${ }^{(25)}$. In 1983, the Joint Expert Committee on Food Additives of the FAO of the WHO established an acceptable daily intake level of $0.7 \mathrm{mg} / \mathrm{kg}$ body weight. A constant fraction of sulphite agents that enter the body via ingestion is metabolised in the liver. However, a finite amount will pass through the organ and enter the systemic circulation. Approximately, $10 \%$ of the ingested dose is excreted unchanged in the urine (26,27). $^{(2)}$.

Another widely used preservative is SB, which is known for its hydroxyl-radical scavenging, bacteriostatic and fungistatic properties under acidic conditions ${ }^{(28)}$. The Joint Expert Committee on Food Additives of the FAO/WHO established an acceptable daily intake level of $5 \mathrm{mg} / \mathrm{kg}$ body weight. However, recent studies have suggested that SB is linked to allergic reactions and, moreover, to hyperactivity in children $^{(29-31)}$. The possible effects of SS and SB on specific metabolic pathways are scarce, albeit SB is a well-documented hydroxyl-radical scavenger and was recently found to suppress Th1-type immune responses in vitro ${ }^{(32,33)}$.

Another compound with a strong antioxidant capacity is curcumin, the major component of turmeric (Curcuma longa). In vitro and animal studies have shown that curcumin exerts various beneficial properties such as anti-inflammatory, anti-neoplastic, anti-cancer and anti-ischaemic effects ${ }^{(34-36)}$. Recently published studies have elucidated the effect of curcumin on metabolisms of obesity and insulin resistance in various animal models. Taken together, these studies demonstrate decreased leptin levels after curcumin consumption in animal models ${ }^{(37-39)}$. In our study, we were able to confirm and to extend these results using a cell culture system of differentiated adipocytes, where we could show a significant decrease in leptin levels already after $12 \mathrm{~h}$ of exposure to curcumin. After an incubation time of $24 \mathrm{~h}$, curcumin also decreased LPS-induced IL-6 levels in the supernatant, which is consistent with the reported anti-inflammatory properties of curcumin ${ }^{(40)}$. However, another possible explanation for the strong effects of curcumin on leptin release could be the induction of apoptosis, as described by various studies $^{(41,42)}$. For SS and SB, mechanisms of apoptosis do not explain the obtained diminution of leptin levels, as IL-6 and nitrite were not affected during the whole experiments.

As we did not observe any effect of the antioxidants on leptin secretion in the absence of LPS, we assumed that the activation of the inducible NO synthase could provide a possible explanation for the affected leptin secretion in LPS-stimulated cells only. Although LPS is well known to activate inducible NO synthase in macrophages and dendritic cells of the skin, thereby elevating concentrations of nitrite, which in turn may affect leptin secretion ${ }^{(43-45)}$, in our model, we did not observe any effect on nitrite release after treatment with LPS alone or in combination with the food additives. These results are in line with previously published studies, which show that only a combination of LPS, TNF- $\alpha$ and interferon- $\gamma$ affects inducible NO synthase activity in murine 3T3-L1 cells, suggesting that the induction of this enzyme is mediated by a complex network of interactions involving inflammatory cytokines and LPS at the level of the adipocytes ${ }^{(46,47)}$.

Taken together, our data suggest a possible effect of the food additives SS, SB and curcumin on the release of leptin from adipocytes in a state of chronic inflammation, which is associated with obesity. Decreased leptin release during the consumption of nutrition-derived food additives would decrease the overall amount of circulating leptin to which the central nervous system is exposed and could thereby influence food intake and contribute to an obesogenic effect ${ }^{(9)}$.

In the murine 3T3-L1 cell culture system, we could analyse the sole effect of the investigated compounds avoiding interfering factors, which affect leptin metabolism, i.e. circulating insulin, glucocorticoids or catecholamines. From the data obtained in the present in vitro study, however, it is unclear how food additives interfere in a complex system such as the human organism with regard to leptin metabolism. Therefore, it is unclear to what extent any conclusion from the present in vitro study can be extrapolated to the in vivo situation, and clearly more studies are needed to investigate the potential contribution of diet-derived agents in a complex organism and a possible influence on the development of obesity.

\section{Acknowledgements}

We highly appreciate the expert technical assistance of Karin Salzmann, Simone Wühl and Astrid Haara. The authors' responsibilities were as follows: C. C. and D. F. conceived and designed the study; C. C. and A. T. were responsible for the data analysis and interpretation; C. C. was responsible for writing the manuscript; M. J., A. T., F. U., M. P., J. P. and C. E. were responsible for the critical revision and its important intellectual content; D. F. was responsible for the study supervision. The authors have nothing to disclose. The present study received no specific grant from any funding agency in the public, commercial or not-for-profit sectors. 


\section{References}

1. Haslam DW \& James WP (2005) Obesity. Lancet 366 , 1197-1209.

2. Maury E \& Brichard SM (2010) Adipokine dysregulation, adipose tissue inflammation and metabolic syndrome. Mol Cell Endocrinol 314, 1-16.

3. Hotamisligil GS, Shargill NS \& Spiegelman BM (1993) Adipose expression of tumor necrosis factor-alpha: direct role in obesity-linked insulin resistance. Science 259, 87-91.

4. Neal MD, Leaphart C, Levy R, et al. (2006) Enterocyte TLR4 mediates phagocytosis and translocation of bacteria across the intestinal barrier. J Immunol 176, 3070-3079.

5. Cani PD, Bibiloni R, Knauf C, et al. (2008) Changes in gut microbiota control metabolic endotoxemia-induced inflammation in high-fat diet-induced obesity and diabetes in mice. Diabetes 57, 1470-1481.

6. Cani PD, Delzenne NM, Amar J, et al. (2008) Role of gut microflora in the development of obesity and insulin resistance following high-fat diet feeding. Pathol Biol (Paris) 56, 305-309.

7. Tilg H \& Moschen AR (2006) Adipocytokines: mediators linking adipose tissue, inflammation and immunity. Nat Rev Immunol 6, 772-783.

8. Zhang Y, Proenca R, Maffei M, et al. (1994) Positional cloning of the mouse obese gene and its human homologue. Nature 372, 425-432.

9. Friedman JM \& Halaas JL (1998) Leptin and the regulation of body weight in mammals. Nature 395, 763-770.

10. Bates SH \& Myers MG Jr (2003) The role of leptin receptor signaling in feeding and neuroendocrine function. Trends Endocrinol Metab 14, 447-452.

11. Ciardi C, Tatarczyk T, Tschoner A, et al. (2010) Effect of postprandial lipemia on plasma concentrations of A-FABP, RBP-4 and visfatin. Nutr Metab Cardiovasc Dis 20, 662-668.

12. Havel PJ, Kasim-Karakas S, Mueller W, et al. (1996) Relationship of plasma leptin to plasma insulin and adiposity in normal weight and overweight women: effects of dietary fat content and sustained weight loss. J Clin Endocrinol Metab 81, 4406-4413.

13. Guerci B, Hadjadj S, Quilliot D, et al. (2000) No acute response of leptin to an oral fat load in obese patients and during circadian rhythm in healthy controls. Eur $J$ Endocrinol 143, 649-655.

14. Despres JP \& Lemieux I (2006) Abdominal obesity and metabolic syndrome. Nature 444, 881-887.

15. Green H \& Kehinde O (1975) An established preadipose cell line and its differentiation in culture. II. Factors affecting the adipose conversion. Cell 5, 19-27.

16. Montague CT, Farooqi IS, Whitehead JP, et al. (1997) Congenital leptin deficiency is associated with severe early-onset obesity in humans. Nature 387, 903-908.

17. Campfield LA, Smith FJ, Guisez Y, et al. (1995) Recombinant mouse $\mathrm{OB}$ protein: evidence for a peripheral signal linking adiposity and central neural networks. Science 269, 546-549.

18. Halaas JL, Gajiwala KS, Maffei M, et al. (1995) Weightreducing effects of the plasma protein encoded by the obese gene. Science 269, 543-546.

19. Pelleymounter MA, Cullen MJ, Baker MB, et al. (1995) Effects of the obese gene product on body weight regulation in ob/ob mice. Science 269, 540-543.

20. Maffei M, Halaas J, Ravussin E, et al. (1995) Leptin levels in human and rodent: measurement of plasma leptin and ob RNA in obese and weight-reduced subjects. Nat Med 1, $1155-1161$.
21. Ostlund RE Jr, Yang JW, Klein S, et al. (1996) Relation between plasma leptin concentration and body fat, gender, diet, age, and metabolic covariates. J Clin Endocrinol Metab 81, 3909-3913.

22. Laimer M, Ebenbichler CF, Kaser S, et al. (2002) Weight loss increases soluble leptin receptor levels and the soluble receptor bound fraction of leptin. Obes Res 10, 597-601.

23. Sandhofer A, Laimer M, Ebenbichler CF, et al. (2003) Soluble leptin receptor and soluble receptor-bound fraction of leptin in the metabolic syndrome. Obes Res 11, 760-768.

24. Fried SK, Ricci MR, Russell CD, et al. (2000) Regulation of leptin production in humans. J Nutr 130, 3127S-3131S.

25. Lester MR (1995) Sulfite sensitivity: significance in human health. J Am Coll Nutr 14, 229-232.

26. Gunnison AF \& Palmes ED (1976) A model for the metabolism of sulfite in mammals. Toxicol Appl Pharmacol 38, 111-126.

27. Gunnison AF \& Jacobsen DW (1987) Sulfite hypersensitivity. A critical review. CRC Crit Rev Toxicol 17, 185-214.

28. Kim SY, Kim CH, Yoo HJ, et al. (1999) Effects of radical scavengers and antioxidant on ischemic acute renal failure in rabbits. Ren Fail 21, 1-11.

29. Nettis E, Colanardi MC, Ferrannini A, et al. (2004) Sodium benzoate-induced repeated episodes of acute urticaria/ angio-oedema: randomized controlled trial. $\mathrm{Br} J$ Dermatol 151, 898-902.

30. Asero R (2006) Sodium benzoate-induced pruritus. Allergy 61, $1240-1241$

31. McCann D, Barrett A, Cooper A, et al. (2007) Food additives and hyperactive behaviour in 3-year-old and 8/9-year-old children in the community: a randomised, double-blinded, placebo-controlled trial. Lancet 370, 1560-1567.

32. Maier E, Kurz K, Jenny M, et al. (2010) Food preservatives sodium benzoate and propionic acid and colorant curcumin suppress Th1-type immune response in vitro. Food Chem Toxicol 48, 1950-1956.

33. De Kimpe SJ, Anggard EE \& Carrier MJ (1998) Reactive oxygen species regulate macrophage scavenger receptor type I, but not type II, in the human monocytic cell line THP-1. Mol Pharmacol 53, 1076-1082.

34. Epstein J, Sanderson IR \& Macdonald TT (2010) Curcumin as a therapeutic agent: the evidence from in vitro, animal and human studies. Br J Nutr 103, 1545-1557.

35. Aggarwal BB, Kumar A \& Bharti AC (2003) Anticancer potential of curcumin: preclinical and clinical studies. Anticancer Res 23, 363-398.

36. Hatcher H, Planalp R, Cho J, et al. (2008) Curcumin: from ancient medicine to current clinical trials. Cell Mol Life Sci 65, 1631-1652.

37. Jang EM, Choi MS, Jung UJ, et al. (2008) Beneficial effects of curcumin on hyperlipidemia and insulin resistance in high-fat-fed hamsters. Metabolism 57, 1576-1583.

38. Seo KI, Choi MS, Jung UJ, et al. (2008) Effect of curcumin supplementation on blood glucose, plasma insulin, and glucose homeostasis related enzyme activities in diabetic $\mathrm{db} / \mathrm{db}$ mice. Mol Nutr Food Res 52, 995-1004.

39. Weisberg SP, Leibel R \& Tortoriello DV (2008) Dietary curcumin significantly improves obesity-associated inflammation and diabetes in mouse models of diabesity. Endocrinology 149, 3549-3558.

40. Menon VP \& Sudheer AR (2007) Antioxidant and antiinflammatory properties of curcumin. Adv Exp Med Biol 595, 105-125.

41. Ejaz A, Wu D, Kwan P, et al. (2009) Curcumin inhibits adipogenesis in 3T3-L1 adipocytes and angiogenesis and obesity in C57/BL mice. J Nutr 139, 919-925. 
42. Kwon Y \& Magnuson BA (2009) Age-related differential responses to curcumin-induced apoptosis during the initiation of colon cancer in rats. Food Chem Toxicol $\mathbf{4 7}$, 377-385.

43. Cruz MT, Duarte CB, Goncalo M, et al. (2001) LPS induction of I kappa B-alpha degradation and iNOS expression in a skin dendritic cell line is prevented by the janus kinase 2 inhibitor, Tyrphostin b42. Nitric Oxide 5, 53-61.

44. Unno Y, Akuta T, Sakamoto Y, et al. (2006) Nitric oxideinduced downregulation of leptin production by 3T3-L1 adipocytes. Nitric Oxide 15, 125-132.
45. Jones E, Adcock IM, Ahmed BY, et al. (2007) Modulation of LPS stimulated NF-kappaB mediated nitric oxide production by PKCepsilon and JAK2 in RAW macrophages. J Inflamm (Lond) 4, 23.

46. Kapur S, Marcotte B \& Marette A (1999) Mechanism of adipose tissue iNOS induction in endotoxemia. Am J Physiol 276, E635-E641.

47. Dobashi K, Asayama K, Nakane T, et al. (2000) Troglitazone inhibits the expression of inducible nitric oxide synthase in adipocytes in vitro and in vivo study in 3T3-L1 cells and Otsuka Long-Evans Tokushima Fatty rats. Life Sci 67, 2093-2101. 